\title{
PERFORMANCE ON CUE RECOGNITION AND EVASIVE ACTION SKILLS AS PREDICTORS OF EFFECTIVE DRIVING IN COLLEGE-AGE DRIVERS
}

\author{
Dong-Yuan Debbie Wang \\ Purdue University \\ West Lafayette, Indiana USA \\ E-mail: wdy92@psych.purdue.edu \\ David F. Pick \\ Purdue University Calumet \\ Hammond, Indiana USA \\ Robert W. Proctor \\ Purdue University \\ West Lafayette, Indiana USA
}

\begin{abstract}
Summary: Two experiments compared self-reported driving effectiveness of licensed drivers (mean age 19 years) to their performance on two simulated driving tasks. For both experiments, drivers first completed a driving history questionnaire. In Experiment 1, they then performed Cue Recognition, which uses stationary line drawings of vehicles as stimuli and requires a turning or braking response to an appropriate stimulus. Males responded faster than females, especially for the most complex choice responses, and reported more tickets. Drivers reporting no tickets responded slower than those reporting at least one ticket, and they reported fewer accidents. In Experiment 2, drivers also performed Evasive Action Skills, which uses more realistic recorded driving scenarios in which the appearance of a hazard is the imperative stimulus that commands the appropriate turn or brake response. Number of errors on Evasive Action Skills correlated significantly with number of self-reported accidents. Response times on Cue Recognition and Evasive Action Skills were correlated, but there was no relation between response times on Cue Recognition and errors on Evasive Action Skills. However, a comparison of the 10 fastest and 10 slowest drivers on Cue Recognition showed that the fastest responders committed significantly more errors on Evasive Action Skills than did the slowest responders. The data in both experiments reflect a speed-accuracy tradeoff.
\end{abstract}

\section{INTRODUCTION}

The attempt to use reaction time (RT) to predict driving performance has a long history (Desilva, 1936; Greenshields, 1936; Lahy, 1927; Miles \& Vincent, 1934). Shorter RT has been associated with better driving performance (Pen, He, Chen, \& Xiao, 2000), both by itself and when it was included in a battery of other measures (Forbes, 1938; Liu, Guan, Huang, \& Zhang, 1997). Long RT has been reported as a characteristic of young, repeat traffic-law offenders (Selling, 1942).

Doron Precision Systems, Inc., a manufacturer of driving simulators for driver training, developed a screening test called Cue Recognition designed to measure the speed with which a 
driver can recognize and react appropriately to a number of threatening traffic cues. We conducted two experiments on college freshmen designed to investigate the validity of using RT and response accuracy on Cue Recognition as a measure of driver ability for predicting both real world and simulated driving performance.

\section{EXPERIMENT 1}

\section{Method}

A total of 121 Purdue University undergraduate drivers (49 males and 72 females) participated. The mean age of the drivers was 18.85 years. The experiment was conducted using a Doron L-300 series driver analyzer simulator, consisting of a computerized control console, a driver's cab, a laser disk player, and a visual projection system. The screen on which the images were projected was $96 \mathrm{~cm}$ x $208 \mathrm{~cm}$ and was located $259 \mathrm{~cm}$ from the cab’s steering wheel.

The Cue Recognition test uses stationary, abstract depictions of vehicles as stimuli, and requires a turning or braking response to an appropriate stimulus. It consists of three parts of eight trials each. Every trial begins with the presentation on the screen of a display containing three to eight symbols representing motor vehicles. Each symbol is approximately $18.5 \mathrm{~cm}$ wide and $13.5 \mathrm{~cm}$ tall at the projection distance used. The number of symbols changes randomly between trials. Initially, all symbols are non-threatening stylized portrayals of a vehicle traveling away from the driver, with no more than one symbol in each of eight equal-sized screen sectors. In the course of a trial the position of the symbols changes every $2 \mathrm{~s}$, effectively changing the scene, but the number of symbols is held constant within a trial. At some time after three to nine scene changes, an action cue replaces one of the non-threatening symbols, signaling the driver to make the appropriate evasive response. In Part I, the action cue is a symbol of a vehicle moving toward the driver, and the appropriate response is to brake as quickly as possible. In Part II, the action cue is a symbol of a vehicle approaching from either the left or right, and the appropriate response is to turn as quickly as possible to the corresponding direction. Part III is a combination of the other parts in which both types of action cues are presented and command the same responses. In addition to performing on Cue Recognition, drivers completed a driving history questionnaire that requested information such as number of tickets, number of miles driven per year, etc.

\section{Results and Discussion}

Males responded faster than females $(t=3.67, p<.001, M \mathrm{~s}=872 \mathrm{~ms}$ for males and $966 \mathrm{~ms}$ for females). This difference was most pronounced in Part II $(t=4.82, p<.001, M s=778 \mathrm{~ms}$ for males and $916 \mathrm{~ms}$ for females) and Part III $(t=3.00, p<.005, M s=959 \mathrm{~ms}$ for males and 1056 ms for females), in which a choice among actions was required. Males also tended to respond faster than females in Part I $(t=1.31, p=.19, M s=878 \mathrm{~ms}$ for males and $928 \mathrm{~ms}$ for females), for which there was only one possible action. Females reported having been issued fewer tickets than males ( $t=2.88, p=.005, M s=1.12$ tickets for males and 0.49 tickets for females). However, this does not necessarily imply that female drivers are more law abiding than male drivers because the males reported driving almost twice as far per year as the females $(t=3.8, p$ $<.005, M s=17,850 \mathrm{~km} /$ year for males and 9,849 km /year for females). 
There were significant differences in RT for drivers grouped on the basis of whether they reported having been issued a ticket for a moving violation. Overall average RT was longer for drivers without tickets $(M=966 \mathrm{~ms})$ than for those with tickets $(M=879 \mathrm{~ms}), t=3.39, p<.005$. This difference was significant in Part I (Ms $=965$ and $833 \mathrm{~ms}$, respectively, $t=3.73, p<.005)$ and Part III (Ms = 1,058 and 963 ms, respectively, $t=2.98, p=.003)$, but not in Part II (Ms = 875 and $840 \mathrm{~ms}$, respectively, $t=1.14, p=.26)$. The drivers with no tickets reported fewer accidents than the drivers with at least one ticket $(M s=0.65$ accidents for the drivers without tickets and 1.04 accidents for those with tickets, $t=2.31, p=.023)$. The difference in age between the two groups was also significant, with the drivers without tickets being younger $(M=$ 18.8 years) than the drivers with at least one ticket $(M=20.1$ years $), t=2.61, p=.01$. These findings are not too surprising because an accident is often accompanied by a citation for a moving violation and older drivers have had more opportunities for being issued a ticket. Correlations between RTs in all three parts of Cue Recognition and the age of the driver were conducted. There were no significant relationships with age ( $r s<.2, p s>.1)$. The age range of drivers is likely too narrow to allow detection of age differences in RT.

Knowing a driver's performance on Cue Recognition does not allow prediction of successful driving performance, as measured by the driving survey, any better than knowing the gender of the driver. A basic premise of using RT data to predict driving performance is that shorter RT will enable a driver to handle hazardous driving situations better. Our findings are that shorter RTs from college-age drivers are associated with more tickets and being male. It could easily be the case that drivers with relatively long RTs are less likely to place themselves in driving situations where having fast reactions are critical to avoiding a crash. It is also possible, given the variation in the situations under which accidents occur, that Experiment 1 did not allow a sensitive enough assessment of the utility of Cue Recognition for predicting successful driving performance. Experiment 2 was conducted in order to reduce the between-subject variability in the situations in which crashes might occur in an attempt to increase the sensitivity of our measures.

\section{EXPERIMENT 2}

\section{Method}

Sixty-three Purdue undergraduate drivers (48 males, 15 females, mean age = 19.4 yrs) completed the driving history questionnaire and performed on Cue Recognition, as in Experiment 1. They also performed on Evasive Action Skills, a drivers' education video from Doron that measures RT for 10 trials of realistic filmed driving scenarios with hazards coming from both sides of the driver, as well as from directly ahead. The appearance of a hazard is the imperative stimulus that commands the appropriate turn or brake response. An error on Evasive Action Skills is equivalent to a simulated crash. The screen size employed by Evasive Action Skills was $86 \mathrm{~cm} \mathrm{x}$ $190 \mathrm{~cm}$. The viewing distance was the same as in Experiment 1. 


\section{Results and Discussion}

There was a positive correlation between overall mean RT in Cue Recognition and overall mean RT in Evasive Action Skills $(r=0.358, p=0.004)$. Although short RT in Cue Recognition was associated with short RT in Evasive Action Skills, there was no statistically significant relation between RT on Cue Recognition and the number of errors on Evasive Action Skills. However, all correlations in the correlation matrix involving RT for Cue Recognition (Parts I, II, III, and overall), and number of errors in Evasive Action Skills were negative ( $r$ s = -.04, -.07, -.15, and .09 , respectively, $p s>.24$ ). In other words, shorter RTs in Cue Recognition tended to be associated with more errors on Evasive Action Skills, as well as with faster responding. But, the correlations between performance on Cue Recognition and Evasive Action errors are so small that they cannot be used to predict the likelihood of an accident (an error) in a simulated driving environment with any degree of accuracy.

A possible reason for the absence of a significant correlation between RT as measured in Cue Recognition and errors measured by Evasive Action Skills could be the nature of the errors themselves. Recorded errors included the kinds of errors that might result in a collision, for example, making the wrong decision in a hazardous situation and not recognizing a hazard in time to evade it. But recorded errors also included failure to follow instructions, for example, not depressing the accelerator or centering the steering wheel. The requirement that the steering wheel be centered was a particular source of error because drivers had a tendency to turn the wheel along with the driving scene. If the wheel was not in the centered position at the start of a timed interval, an error was generated. In short, the error data from Evasive Action Skills includes random variation, which reduces the sensitivity of any statistical analysis.

For a more sensitive analysis, the 10 drivers with the shortest overall RTs on Cue Recognition and the 10 drivers with the longest overall RTs were compared on performance on Evasive Action Skills. Although the difference in number of errors recorded for the two groups was large ( $M=3.1$ for the fastest group, $M=2.0$ for the slowest group), it was not statistically significant ( $t$ $=1.49, p=.153)$. We then formed two groups of 10 drivers based on the shortest and longest mean RTs on Part III of Cue Recognition, the most difficult of the three parts. The group responding the fastest on Part III committed significantly more errors on Evasive Action Skills $(M=3.7)$ than did the group responding the slowest $(M=1.8), t=2.61, p=.018$. A consistent theme running through our data is that faster responding is associated with an increase in errors. In other words, individuals who respond fast on Cue Recognition tend to make more errors on the simulated driving involved in Evasive Action Skills.

Performance on Cue Recognition did not correlate with self-reported accident rates from the driving history questionnaire $(r=-.04, p=.78)$. However, there was a significant correlation between the number of reported accidents and number of errors on Evasive Action Skills ( $r=$ $0.25, p=.047$ ), but not between the number of tickets reported and the number of errors on Evasive Action Skills ( $r=0.03, p=.818$ ). Thus, performance on Evasive Action Skills was a better predictor of number of reported accidents than was performance on Cue Recognition. 


\section{CONCLUSION}

RTs on Cue Recognition correlate with some aspects of reported driving problems and of performance in simulated driving, specifically number of tickets received, speed of making evasive actions, and number of incorrect evasive actions. The apparent relation is that people in this age group who tend to respond fast also tend to have more driving problems, both in the simulated environment and the real world. In other words, fast responding reflects a bias to respond quickly at the expense of accuracy, rather than a better driving ability. The only significant correlation reported between number of accidents and performance on the two tasks was number of errors on Evasive Action Skills. Because the more complicated scenarios in Evasive Action Skills are more comparable to those encountered in driving than those in Cue Recognition, it may be that performance in simulated driving scenarios is a better predictor of likelihood of accident in real driving than is performance on more artificial tasks.

\section{ACKNOWLEDGEMENTS}

We would like to thank Doron Precision Systems, Inc., and Deborah Quackenbush for providing the driving simulator and scenarios used for the experiments.

\section{REFERENCES} 10, 1-13.

Desilva, H. R. (1936). On an investigation of driving skill. Human Factors (London),

Forbes, T. W. (1938). Age performance relationships among accident-repeater automobile drivers. Journal of Consulting Psychology, 2, 143-148.

Greenshields, B. D. (1936). Reaction time in automobile driving. Journal of Applied Psychology, 20, 353-358.

Lahy, J. M. (1927). La section psycho-physiologique des travailleurs: conducteurs de tramways et d'autobus (The psycho-physiological selection of workers: conductors of tramways and autobuses). Paris: Dunod, 240.

Liu, Z., Guan, L., Huang, Y., \& Zhang, Z. (1997). Compilation and application of the occupation suitability test for military motor drivers. Psychological Science (China), 18, 242245.

Miles, G. H., \& Vincent, D. F. (1934). The institute's tests for motor drivers. Human Factors (London), 8, 245-257.

Pen, C., He, C., Chen, B., \& Xiao, J. (2000). A comparative study of reaction time between accident repeaters and safe truck drivers. Psychological Science (China), 23, 203-206.

Selling, L.S. (1942). The young traffic offender. American Journal of Orthopsychiatry, 12, 241-251. 